\title{
Evaluation of Timetable of the Second Permanent Molar Calcification among Children between 5 and 17 Years of Age
}

\author{
AHMAD JAFARI ${ }^{1,2}$, ABBAS TAHER ${ }^{2,3,4}$, MOHAMMAD REZA KHAMI ${ }^{1}$, MARYAM SHAKUR SHAHABI ${ }^{5}$, AHMAD REZA \\ SHAMSHIRI ${ }^{1}$, FARID BARADARAN NAKHJAVANI ${ }^{1}$, MAJID MEHRAN ${ }^{6}$ \\ ${ }^{1}$ School of Dentistry, Tehran University of Medical Sciences, Tehran, Iran. Email: ajafari@tums.ac.ir \\ ${ }^{2}$ School of Dentistry, Al Hussain University College, Karbala, Iraq. Email: kufa54@gmail.com \\ ${ }^{3}$ The Islamic University Najaf, Najaf, Iraq.Email: mkhami@tums.ac.ir \\ ${ }^{4}$ Visiting Professor, School of Dentistry, Tehran University of Medical Sciences, Tehran, Iran \\ ${ }^{5}$ School of Dentistry, Zanjan University of Medical Sciences, Zanjan, Iran. Email: maryam.shahabi@gmail.com \\ ${ }^{6}$ School of Dentistry, Shahed University, Tehran, Iran.Email: m.mehran@shahed.ac.ir \\ Corresponding author: Majid Mehran, Department of Pediatric Dentistry, School of Dentistry, Shahed University, Tehran, Iran \\ Address: School of Dentistry, Shahed University, Italia St., Ghods St, Enghelab Blvd., Tehran, Iran. Tel: +98- 21- 8895 9210, Fax: +98- 21- \\ 8896 7618, Cell phone: +98-912-175 5744, E-mail:m.mehran@shahed.ac.ir
}

\begin{abstract}
Objective: This study evaluates timetable of calcification stages of the second permanent molars (SPMs) based on panoramic radiographic findings from a selected group of Iranian patients.

Methods: This descriptive, analytical and cross sectional study uses 715 panoramic radiographs of subjects with optimal quality between 5 to 17 years of age ( $n=60$ for each age group) whom were selected from the referrals to the departments of Orthodontics and Pediatric Dentistry of at Tehran University of Medical Sciences, Iran. Patients with systemic diseases or any kind of syndromes were excluded. Calcification stages were determined based on Demirjian's classification. Data were analyzed using generalized estimating equation (GEE).

Results: Only $20 \%$ of SPMs of 5 year-olds were in their $2^{\text {nd }}$ stage of calcification and the remaining showed higher stages. The mean age of crown completion was around 8.5 years while the mean ages of root completion for the open and closed apices were 13 years and 3 months and 15.5 years, respectively. Also calcification occurred at earlier ages among girls.

Conclusion: Timetable of calcification of SPMs is useful to predict the onset of puberty. Demirjian's classification well matches Iranian population for determining calcification stage of SMPs.

Keywords: Permanent Molar, Tooth Calcification, Forensic Dentistry, Iranian Population

Key Messages: Mean age at different stages of calcification of the second permanent molars could be effective in forensic dentistry as well as for diagnostic and treatment purposes.
\end{abstract}

\section{INTRODUCTION}

Age estimation is routinely used in forensic medicine as a legal index. The calcification timetable of second permanent molars (SPMs) can be a useful characteristic for many dentistry, anthropology, and archeology purposes. It is well known that development and eruption of teeth is a reliable index for the estimation of chronological age. ${ }^{1,2}$ Calcification stages of canines, premolars and the second molars is well matched with skeletal maturity however it is believed that the developmental stage of second molars has the highest correlation in that regard which allows clinicians to estimate puberty by assessing its calcification stage using panoramic radiographs ${ }^{3-5}$

Evidence shows that accelerated or delayed eruption of SPMs can be anticipated in different classes of occlusion, which is important in orthodontic treatments for instance, the shortage of space for the eruption of mandibular SPMs is usually seen in Class II people. ${ }^{4}$

Also in dental practice, calcification stage of SMPs is an important prognostic measure when the first permanent molars (FPMs) are suggested to be extracted. For example, treatment of FPMs with extensive caries is a challenging event for the child and the clinician; especially when the pulp is also involved and prognosis is often poor in such cases so extraction of FMPs might be of better indication. ${ }^{6,7}$ It has been shown that if SPM is in optimal calcification stage then closure of hallow space after extraction of FPMs needs no further clinical intervention. ${ }^{8}$
Therefore the calcification status of the SPM is important for clinical decision making. If FPM is lost at older ages when the majority of root length of SPM is already formed, then no longer rotational movement occurs. This phenomenon, especially in the mandible may cause many problems including incomplete space closure of the lost FPM. $^{9}$

In this study we assess the mean age of different stages of calcification onset of SPM using panoramic radiographs from an Iranian population.

\section{SUBJECTS AND METHODS}

This is a descriptive, analytical and cross-sectional study on panoramic radiographs selected from the archives of Department of Orthodontics and Pediatric Dentistry of Tehran University of Medical Sciences, Tehran, Iran. Selected subjects were at the ages ranging from 5 to 17 years old. Sixty radiographs of each age (a total of 715) were selected. A questionnaire was used to extract relevant information from patients' files containing demographic, age and gender details. Presence of systemic disease was an exclusion criterion. Then the calcification stage of the SPMs was assessed using Demirjian's classification ${ }^{10}$ (Figure 1). 


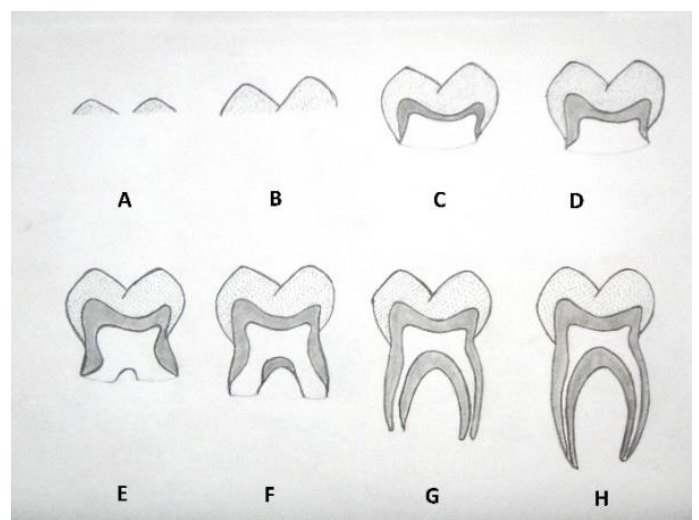

Figure 1. Stages of calcification of SPMs according to Demirjian's classification

Panoramic radiographs of optimal quality were selected and then two examiners with sufficient clinical experience for interpretation of panoramic radiographs assessed the stage of calcification for SPMs independently. In a case of disagreement on level of calcification between two examiners, a blind third opinion was sought and a conclusion was reached. All three examiners were calibrated for intra-examiner and inter-examiner diagnostic errors in a pilot study. The exclusion criteria was either the presence of any cyst or tumor detectable on the panoramic radiographs around the SPMs or a systemic diseases that could affect the normal process of calcification such as hyperparathyroidism or syndromes causing ectodermal dysplasia on radiographs or in patient's medical file. The Demirjian's classification ${ }^{10}$ for staging SPM calcification comes as bellow:

Stage A. Cusp tips of molar teeth are mineralized but have not yet coalesced.

Stage B. Mineralized cusp tips are united.

Stage C. Calcification of crown is complete.

Stage $D$. The crown formation is completed down to the cementoenamel junction (CEJ) but roots have yet to be formed.

Stage $\mathrm{E}$. The length of the calcified root is shorter than the crown.

Stage $\mathrm{F}$. The length of the calcified root is longer than the crown.

Stage G. Root walls are parallel, but apices remain open.

Stage $\mathrm{H}$. Apical foramen is closed.
Since the Demirjian's classification only evaluates the stages of calcification, we used a stage zero where only a radiolucent mass was seen with no sign of calcification on radiographs.

Analysis of data and all descriptive statistics including frequency, percentage, mean and standard deviation were carried out using SPSS software. We considered a $\mathrm{P}<0.05$ statistically as significant. The correlation between independent variables at different stages of calcification was assessed by generalized estimating equation (GEE) using linear analysis for exchangeable correlation structure.

\section{RESULTS}

715 panoramic radiographs, of them 407 females and 308 males were included in this study. Since no radiograph of children younger than 5 years of age was available, we were unable to identify dental follicle formation or the first stage of calcification according to the Demirjian's classification (initiation of the calcification of cusp tips) In only $20 \%$ of 5 years old children showed mineralized cusp tips of SPMs (second stage) and their remaining teeth were in higher stages of calcification. Number of SPMs at stage 2 decreased when age advanced and number of SPMs at stage 2 dropped to $7.4 \%$ and $4.5 \%$ at the ages of 6 and 7 years, respectively. Thus, we were not able to calculate the mean age for this stage. This problem similarly happened for assessing the mean age for the $3^{\text {rd }}$ stage (crown calcification) also. However, the results showed that $64.5 \%$ of the SPMs at the age of 5 and $76.1 \%$ of them at the age of 6 were in stage C. The frequency of SPMs at this stage (stage $\mathrm{C}$ ) decreased through older ages. SPMs were often at higher stages of calcification among older children. Table 1 shows the number of SPMs at different stages of calcification in each specific age group. The mean value of stage of calcification also was computed based on age, gender and mandible/maxilla to show the correlation between multiple variables (Table 2).

The mean age for completion of crown formation (stage $D$ ) was 8 years and 5 months ( 8.4 years). The mean age for stage $E$ (where the root length is shorter than the crown length) was 10 years and 2 months and the mean age was 13 years and 3 months for stage $G$ (where parallel root walls with an open apex were visible). The mean age for closure of apical foramen of SPMs was 15.5 years (Diagram 1).

Table 1. The frequency of different stages of calcification of SPMs (Demirjian's classification) in each age group

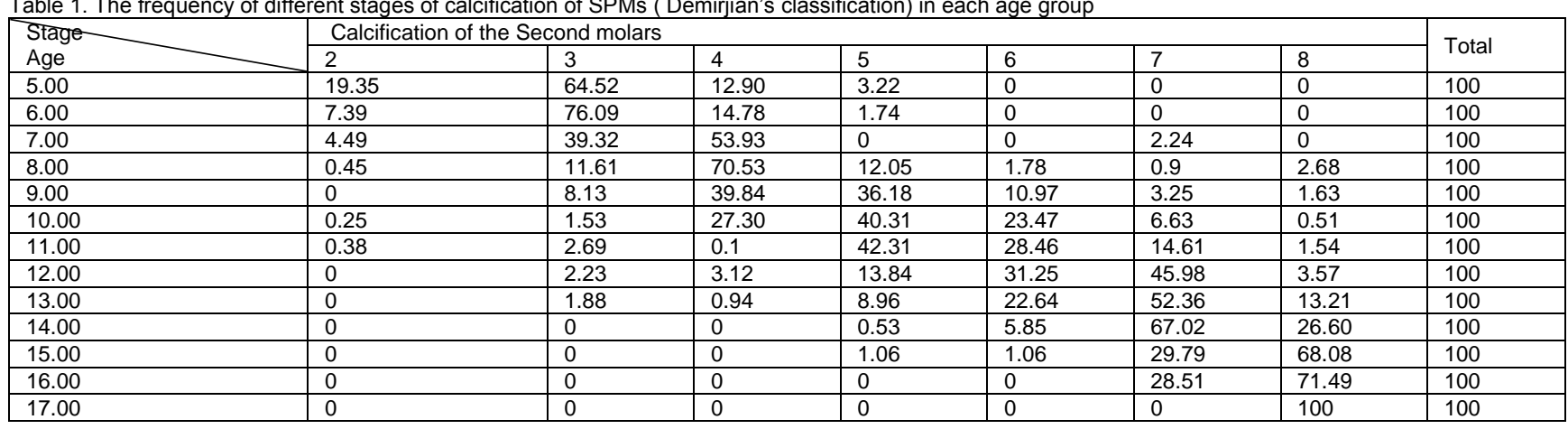


Table 2. The mean value of Demirjian's stage of calcification, according to age, gender and mandible/maxilla.

\begin{tabular}{|l|l|l|l|l|}
\hline \multirow{2}{*}{ Age } & Maxilla & \multicolumn{3}{l|}{ Mandible } \\
\cline { 2 - 5 } & female & male & female & male \\
\hline 5.00 & 3.15 & 2.94 & 3.12 & 2.86 \\
\hline 6.00 & 3.19 & 3.10 & 3.10 & 3.06 \\
\hline 7.00 & 3.58 & 3.72 & 3.40 & 3.60 \\
\hline 8.00 & 4.17 & 4.28 & 4.04 & 4.28 \\
\hline 9.00 & 4.57 & 4.56 & 4.81 & 4.69 \\
\hline
\end{tabular}

\begin{tabular}{|l|l|l|l|l|}
\hline 10.00 & 5.18 & 4.81 & 5.38 & 4.83 \\
\hline 11.00 & 5.62 & 5.21 & 5.68 & 5.29 \\
\hline 12.00 & 6.39 & 6.04 & 6.47 & 6.04 \\
\hline 13.00 & 6.73 & 6.57 & 6.71 & 6.48 \\
\hline 14.00 & 7.29 & 7.13 & 7.27 & 6.94 \\
\hline 15.00 & 7.72 & 7.56 & 7.67 & 7.58 \\
\hline 16.00 & 7.76 & 7.67 & 7.73 & 7.69 \\
\hline 17.00 & 8.00 & 8.00 & 8.00 & 8.00 \\
\hline
\end{tabular}

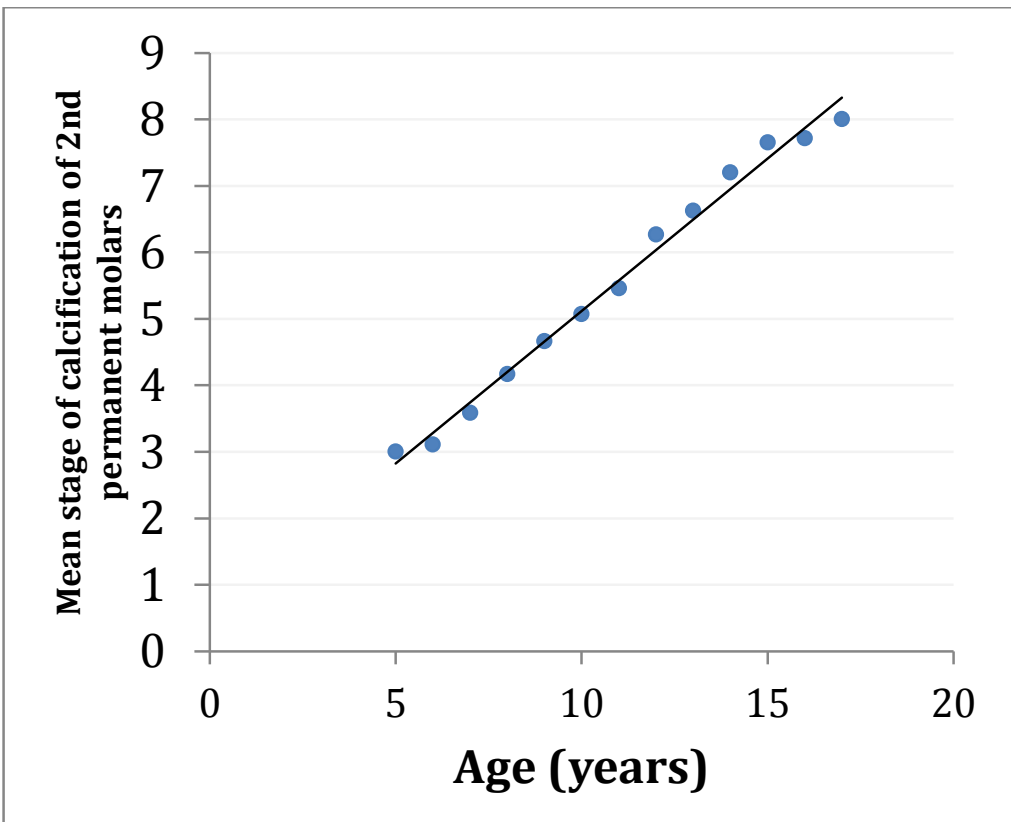

Diagram 1. The mean age for different stages of calcification of SPMs (Y axis represents Demirjian's classification)

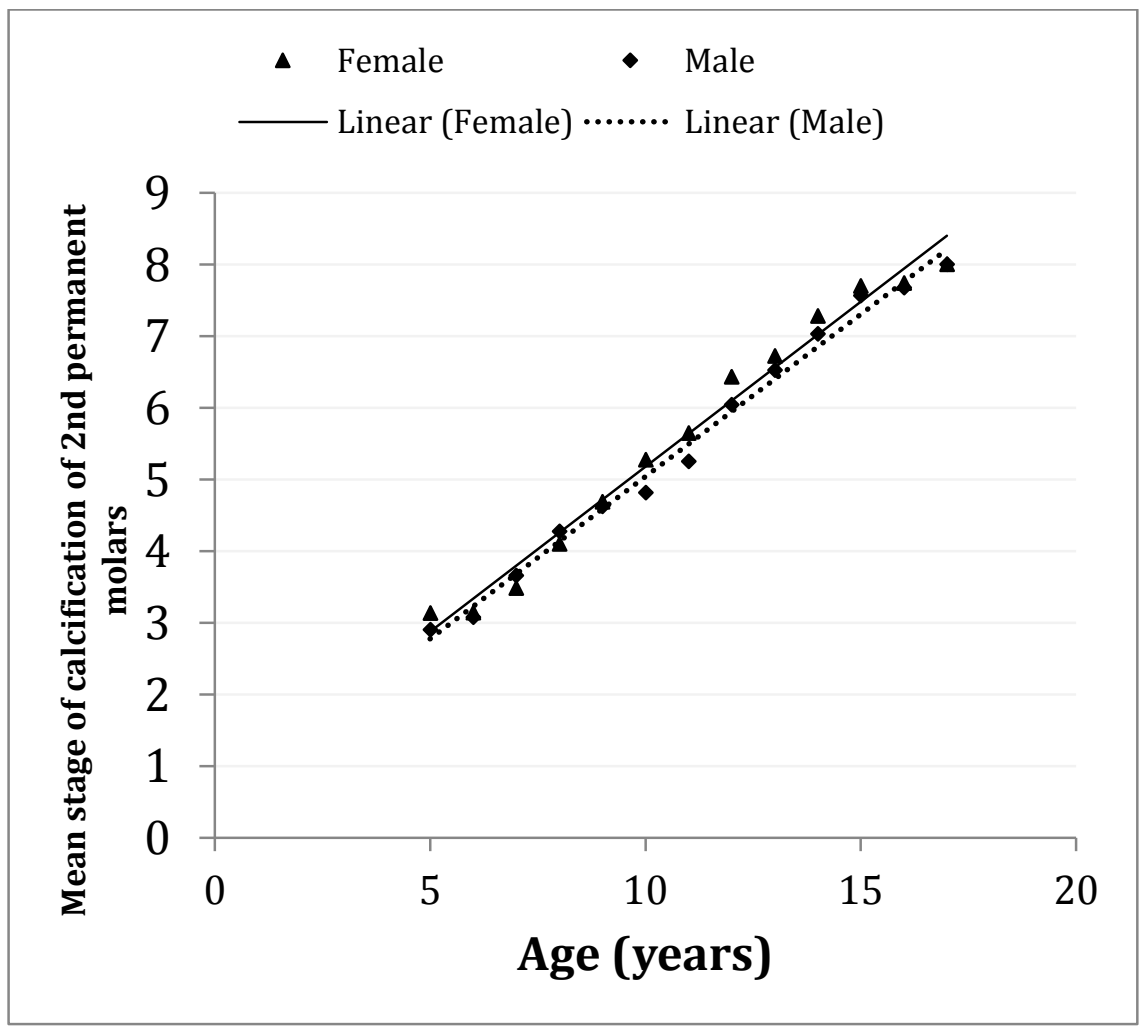


Diagram 2. correlation between gender and stages of calcification of SPMs for different age groups (the $Y$ axis represents the Demirjian's classification)
Diagrams 3 and 4 show calcification age of SPMs among female and male in relation with maxilla and mandible respectively.

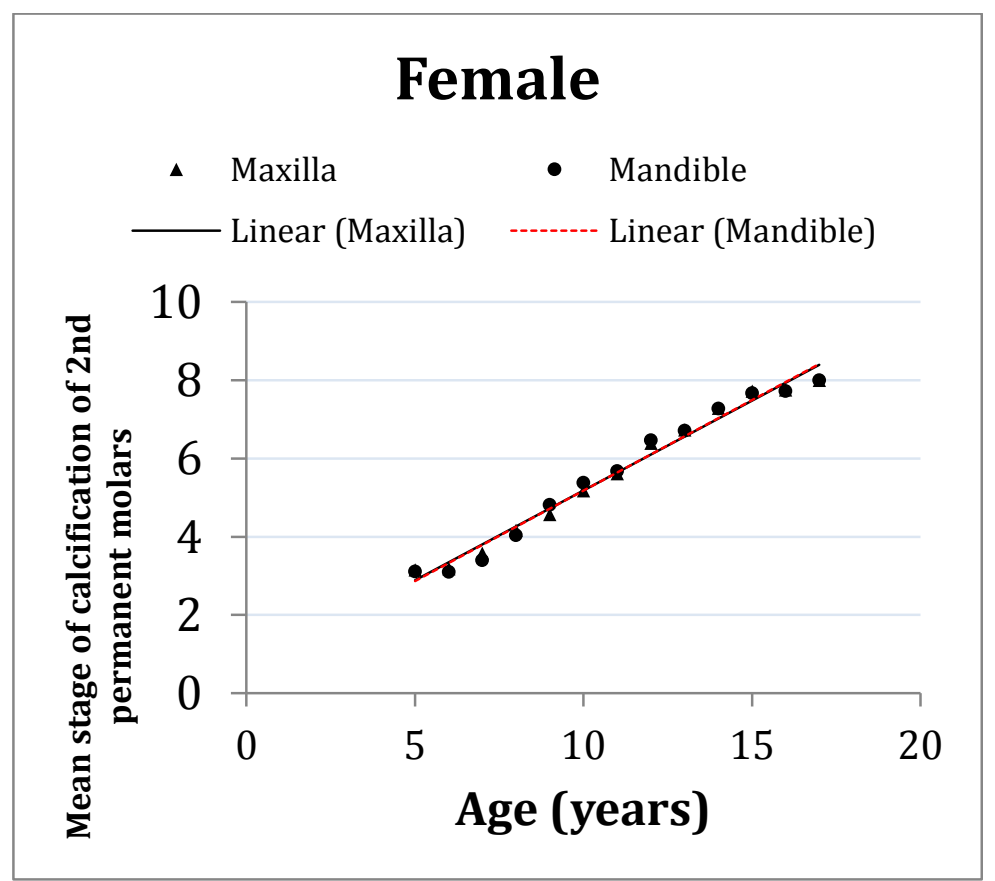

Diagrams 3. Calcification of SPMs among female based on jaws.

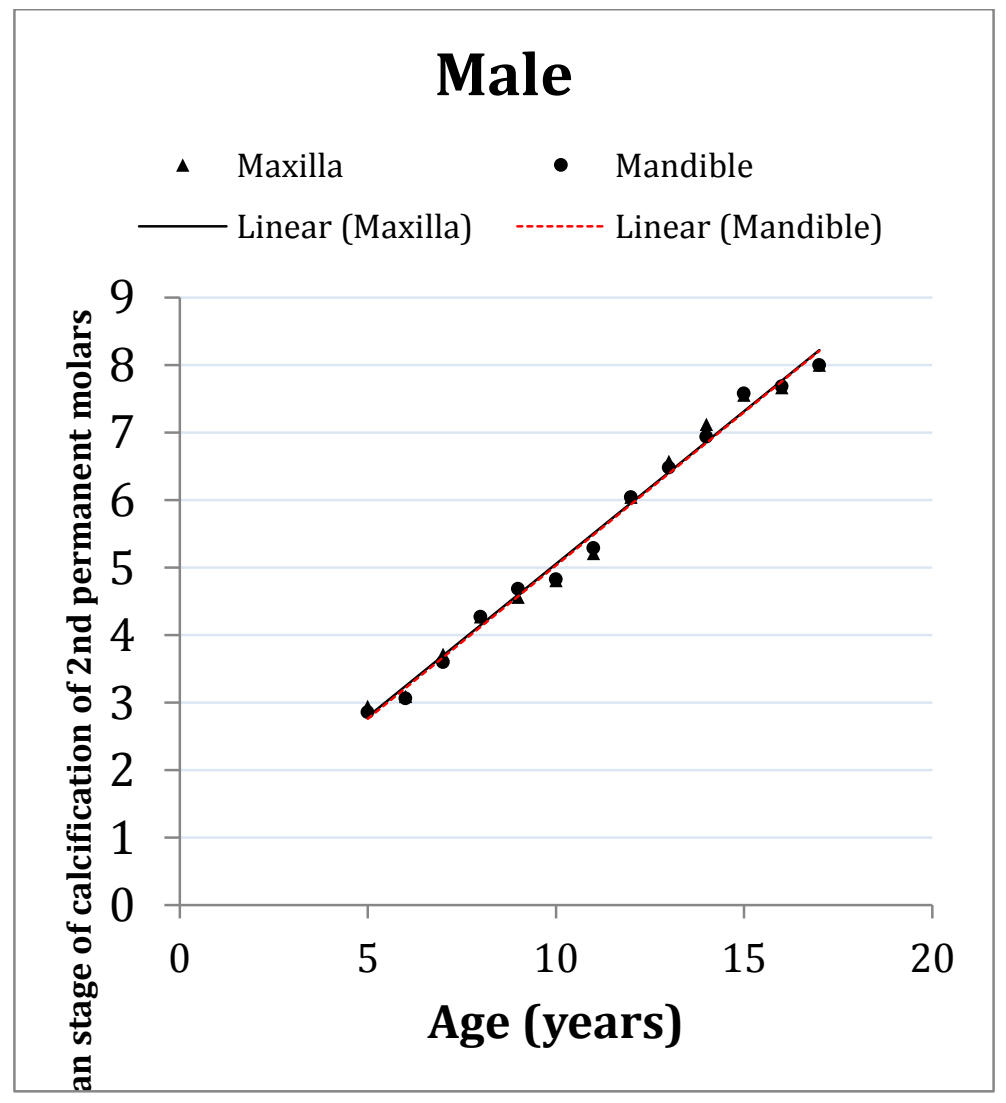

Diagrams 4. Calcification of SPMs among male based on jaws. 
The effect of independent variables on different stages of calcification of SPMs was evaluated by GEE using linear analysis with exchangeable correlation structure results. The effect of gender on different stages of calcification in the maxillary and mandibular jaws was separately computed which showed significant difference in univariate analysis $(P<0.001)$. Calcification occurred at younger ages among girls and at earlier ages for maxilla in comparison with boys and mandible. Since the mean age of female cases was higher than our male cases, all patients were matched for each age group that led to a $\beta$ of 0.87 for gender and 0.12 for the mandible. In the next step, the effect of jaw (maxilla/mandible) on stage of calcification was evaluated after adjusting it for age. Accordingly no correlation was found between the maxilla and the stage of calcification, however gender had a significant correlation with the stage of calcification $(\beta=0.25, \quad P=0.001)$. Calcification of SPMs occurred earlier among girls compared to boys (Diagram 2).

\section{DISCUSSION}

The knowledge of using teeth condition for age estimation has a 170 years of history in medicine. Since then the mean age at different stages of calcification has been broadly used in different disciplines such as forensic science, anthropology and archeology. ${ }^{2}$ However, the modern pediatric dentistry and orthodontics now use this clinical index for diagnostic and treatment purposes in. Finding of an Indian study on third molar eruption which used orthopantomograms showed that calcification stage of SMP could be a significant predictor to assess the chronological age. ${ }^{1}$ We obtained the same results. Ash presented a timetable for the calcification of SPMs that showed mandibular and maxillary SPMs develop approximately at the same time. He mentioned the mean age of 2.5-3 years for the onset of calcification (Demirjian's stage A) and the mean age of 7-8 years for complete calcification of crown (Demirjian's stage D) ${ }^{11}$, however our findings show that the roots of mandibular SPMs are completed slightly earlier than that for their counterparts in the maxilla (14-15 versus 14-16 years). Another study from Washington University claimed that calcification and eruption of maxillary SPMs occurred earlier than the suggested mean age in Class II subjects. ${ }^{12}$ This is also true for the mandibular SPMs of Class III subjects. In other words, increased ANB angle is associated with accelerated calcification and eruption of maxillary SPMs. A study from Tokyo Medical University also demonstrated a delay in eruption of maxillary SPMs in Class III subjects. ${ }^{13}$ However, this difference in eruption for other teeth was not significant. In addition to these studies, another study reported similar stages of development of SPMs in Angle's Class II and III subjects. ${ }^{14}$

There are couple of studies conducted by Mashhad University of Medical Sciences on children, which showed no significant difference between boys and girls in terms of SPM root formation. They also found no difference in calcification between the maxilla and mandible in boys or girls. ${ }^{15,} 16$ In contrast to our findings there is a study from India that showed higher stages of calcification of SPMs in males compared to females. ${ }^{5}$ Another study conducted in South Korea on the stages of calcification and eruption of SPMs showed that in patients with retruded maxilla, the maxillary SPMs had delayed calcification and eruption compared to the mandibular SPMs. In that regard some other researchers have claimed that eruption of SPMs started after formation of 1/4 of the root (Demirjian's stage $\mathrm{F})$, and it took 2-3 years to reach the alveolar bone surface and at this time, $2 / 3$ of the roots were already formed. ${ }^{17-19}$ In another study from South Korea, initiation of root calcification was seen to be at around 8 years of age while they found no significant difference in stages of calcification between two genders or between the maxilla and mandible. However, they found that root calcification occurred earlier in mandibular SPMs compared to the maxillary SPMs in Class III subjects. ${ }^{20}$

Overall our study showed some differences in terms of the effect of gender and type of the jaw (maxilla/mandible) on the calcification stages of SPMs; which may be attributed to the effect of some confounding factors which has been found in studies ${ }^{21}$; such as genetics, hormonal, nutritional, environmental, gender and ethnicity factors that might had an impact on tooth development.

\section{CONCLUSION}

We believe that the timetable of calcification of SPM is useful in dental clinics. It helps clinicians having a proper treatment plan especially when first permanent molar is candidate to be extracted in children due to an excessive caries and pulp involvement. Since, it is suggested that the geographical parameters may affect this timetable according to population diversity, genetic background and also oral health status, so clinicians must consider it for orthodontic treatment.

Clinical relevance: If the first permanent molar has poor prognosis based on extended caries and has no chance to be saved, it would become a candidate for extraction. Based on this study, the best time of extracting this type of tooth is after approximately 8.5 years of age when the root calcification starts, which in turn protects the crown of the second permanent molar from rotational movement. Also, it should be extracted no longer than the 10th year when half of roots are calcified, since after this time the bodily movement of second permanent molar changes to tilting.

Acknowledgement: The authors would like to thank Dr. Flora Elhami, Dr. Mojgan Naseh and Mrs. Nahid Bagheri for their sincere cooperation in the conduction of this study.

Statement of author contributions:

Ahmad Jafari helped shape the research and supervised the project and collected data.

Abbas Taher took the lead in writing the manuscript.

Mohammad Reza Khami conceived of the presented idea and planned the project.

Maryam Shakur Shahabi planned the project and collected data. Ahmad Reza Shamshiri analyzed the data and contributed to the interpretation of the results.

Farid Baradaran Nakhjavani contributed to sample preparation. Majid Mehran contributed to finalize and manage the publication of the article. 


\section{REFERENCES}

1. Tuteja, Monica; Bahirwani, Shraddha; P., Balaji. An evaluation of third molar eruption for assessment of chronologic age: A panoramic study. Journal of Forensic Dental Sciences;Jan-Jun2012, Vol. 4 Issue 1, p13

2. Manjunatha B S, Soni Nishit K. Estimation of age from development and eruption of teeth. J Forensic Dent Sci. 2014 May-Aug; 6(2): 73-76.

3. Suleekorn Krailassiri, Niwat Anuwongnukroh, Surachai Dechkunakorn. Relationships between dental calcification stages and skeletal maturity indicators in Thai individuals. Angle Orthod 2002;72(2):155-66

4. Majourau A, Norton LA. Uprighting impacted second molars with segmented springs. Am J Orthod Dentofacial Orthop 1995;107:235-238.

5. B Rai, S Anand. Relationship of Hand wrist and panoramic radiographs. The Internet Journal of Forensic Science. 2007 Volume 3 Number 1.

6. Penchas $\mathrm{J}$, Peretz $\mathrm{B}$, Becker $\mathrm{A}$. The dilemma of treating severely decayed first permanent molars in children: to restore or to extract. ASDC J Dent Child 1994;61:199-205,.

7. Crabb J, Rock W. Treatment planning in relation to the first permanent molar. Br Dent J 1971 November;131:396-401.

8. Birgitta Jalevik, Marie Moller. Evaluation of spontaneous space closure and development of permanent dentition after extraction of hypomineralized permanent first molars. Int $\mathrm{J}$ Paediatr Dent 2007;17(5):328-35

9. Nikolaos Gkantidis, Simeon Psomiadis, Nikolaos Topouzelis. Teeth spacing: etiology and treatment. Hel Orthod Rev 2007; 10(2):75-92

10. Demirjian A, Goldstein H, Tanner JM. A new system of dental age assess- ment, Human Biology 45 (1973) 211 227.

11. Ash, Major M, Nelson, Stanley J. Wheeler's dental anatomy, physiology, and occlusion. Philadelphia: W.B. Saunders. 2003; pp. 32, 45, and 53.
12. Haruki $T$, Kanomi $R$, Shimono $T$. The differences in the chronology and calcification of second molars between angle Class III and Class II occlusions in Japanese children. ASDC J Dent Child 1997;64(6):400-4.

13. Szilvia Arany, Mitsuyoshi lino, and Naofumi Yoshioka, Radiographic survey of third molar development in relation to chronological age among Japanese juveniles. J Forensic Sci 2004;49(3):534-8.

14. Ilana Brina, Semin Camasuvib, Nasser Dalic, Dror Aizenbudd. Comparison of second molar eruption patterns in patients with skeletal Class II and skeletal Class I malocclusions, Am J Orthod Dentofacial Orthop 2006;130(6):746-751.

15. Heravi F, Ghasemi M, Shanazary M. A comparative study between pattern of eruption of second molars in class II and class III malocclusion with class I. Journal of Dentistry. Mashhad University of Medical Sciences 2006;30:319-26.

16. Ajami B. Imanimoghaddam M. Imen Shahidi M. Radiogrphic Evaluation of Third Molar Developmental Stages in A Group of Iranian Children and Adolescents. Shiraz Univ Dent J 2007;15(2):1-9

17. Garn, S, Lewis A, Koski $P$. The sex difference in tooth calcification, J Dent Res 1958;37:561-7.

18. Shumakar D, Hadary M El. Roentogenographic study of eruption. JADA 1960;61:535-541.

19. Demirjian, A, Buschang $P$, Tanguay $R$, Patterson D. Interrelationships among measures of somatic, skeletal, dental and sexual maturity. Am J Orthod 1985;88:433-438.

20. Cha KS. A study on calcification of the second molars in skeletal Class III malocclusions. Korean J Orthod 1981;11(2):101-108

21. Bayerlein P, Allegrini T. Morphological and clinical consideration of first and second permanent molar eruption disorder. Ann Anat 2006;188(4):353-361. 